MATEC Web of Conferences 13, 04024 (2014)

DOI: $10.1051 /$ matecconf/ 20141304024

(C) Owned by the authors, published by EDP Sciences, 2014

\title{
Electrodeposition of Iridium Oxide by Cyclic Voltammetry: Application of Response Surface Methodology
}

\author{
Saeid Kakooei ${ }^{\text {a, }}$, Mokhtar Che Ismaila, Bambang Ari-Wahjoedia \\ a Centre for Corrosion Research, Department of Mechanical Engineering, Universiti \\ Teknologi PETRONAS, Tronoh31750, Malaysia
}

\begin{abstract}
The effects of scan rate, temperature, and number of cycles on the coating thickness of IrOx electrodeposited on a stainless steel substrate by cyclic voltammetry were investigated in a statistical system. The central composite design, combined with response surface methodology, was used to study condition of electrodeposition. All fabricated electrodes were characterized using electrochemical methods. Field emission scanning electron microscopy and energy-dispersive X-ray spectroscopy were performed for $\mathrm{IrO}_{\mathrm{x}}$ film characterization. Results showed that scan rate significantly affects the thickness of the electrodeposited layer. Also, the number of cycles has a greater effect than temperature on the $\mathrm{IrO}_{\mathrm{X}}$ thickness.
\end{abstract}

\section{Introduction}

Measurement of solution $\mathrm{pH}$ is important and critical in various industries. Classic glass electrode $\mathrm{pH}$ probes exhibit several disadvantages, including instability at high temperatures and mechanical fragility [1]. Alternatively, solid-state $\mathrm{pH}$ sensors can be used as glass $\mathrm{pH}$ sensors. $\mathrm{IrO}_{\mathrm{X}}$ is one of the best $\mathrm{pH}$-sensitive materials given its robust shape, fast response, and high stability over a wide range of pressure and temperature [2,3]. Techniques in fabricating $\mathrm{IrO}_{\mathrm{X}} \mathrm{pH}$ sensors include (i) sputtering , (ii) the sol-gel process, (iii) thermal oxidation of iridium, and (iv) electrodeposition. Among these methods, electrodeposition is considered the most advantageous because it can be performed using a low-cost fabrication process, requires low temperature, uses an affordable substrate, and allows versatility in the use of sensor shapes [4,5].

Optimization techniques such as response surface methodology (RSM) has recently been applied to optimize electrodeposition involving various alloys. However, no statistical studies have been reported on the fabrication of an $\mathrm{IrO}_{\mathrm{x}} \mathrm{pH}$ sensor by electrodeposition. The use of RSM in statistical analysis elucidates the aforementioned process. RSM also decreases the number of required experimental runs to create statistically valid results and saves time and resources. The first step in RSM involves the design of experiments (DOE), which determines the number of experiments that provide reliable and acceptable measurements of the chosen response. Central composite design (CCD) can be employed by RSM as an experimental design to fit a model by the least-squares technique. CCD requires a minimum number of experiments as the standard $2 \mathrm{n}$ factorial with its origin at the centre and $2 \mathrm{n}$ axial points fixed axially at a distance, say $\alpha$, from the centre and replicate

*Corresponding Author: saeid.k_g01663@utp.edu.my, skakooei59@hotmail.com

This is an Open Access article distributed under the terms of the Creative Commons Attribution License 2.0, which permits unrestricted use, distribution, and reproduction in any medium, provided the original work is properly cited. 
experiments at the centre. RSM helps investigate the effects of interactions among independent variables on a selected response.

\section{Materials and methods}

Iridium (IV) chloride hydrate, oxalic acid, potassium carbonate (anhydrous), 30\% hydrogen peroxide, and universal $\mathrm{pH}$ buffers (4, 7, and 9) were purchased from Sigma-Aldrich (USA). All chemicals were used without further purification. A type-316 stainless steel was used as a substrate, and an $\mathrm{Ag} / \mathrm{AgCl}$ reference electrode was used with the $\mathrm{pH}$ sensor for potential recording.

Table 1. Codified and normal values of the experimental design levels

\begin{tabular}{|c|c|c|c|c|c|}
\hline \multicolumn{2}{|c|}{ Parameters } & \multicolumn{5}{|c|}{ Levels } \\
\cline { 2 - 6 } & -1 & -0.5 & 0 & 0.5 & 1 \\
\hline Scan Rate $(\mathrm{mV} / \mathrm{s}): \mathrm{A}$ & 50 & 87.5 & 125 & 162.5 & 200 \\
\hline Temperature $\left({ }^{\circ} \mathrm{C}\right): \mathrm{B}$ & 25 & 35 & 45 & 55 & 65 \\
\hline Cycles (cycle) :C & 100 & 200 & 300 & 400 & 500 \\
\hline
\end{tabular}

Stainless steel electrodes were fabricated as described in our previous work [10]. A highperformance potentiostat/galvanostat (Autolab/PGSTAT128N, the Netherlands) was used for electrodeposition and electrochemical experiments. $\mathrm{CV}$ was applied for electrodeposition in a potential range of $-0.5 \mathrm{~V}$ to $0.65 \mathrm{~V}$ versus $\mathrm{Ag} / \mathrm{AgCl}$ at various scan rates, temperatures, and cycles, as indicated in Table 1.

An electrodeposition solution was prepared as mentioned by Yamanaka [6]. Electrochemical impedance spectroscopy (EIS) measurements were conducted using an Autolab/PGSTAT128N potentiostat. The frequency used for the impedance measurements ranged from $0.01 \mathrm{~Hz}$ to $10 \mathrm{kHz}$. NOVA-1.8 was used for the EIS measurements and curve fitting analysis. A field-emission scanning electron microscope (HITACHI) and an EDX quantitative energy dispersive X-ray analyser were used to examine the characteristics of the electrode surface.

\section{Design of experiments, analysis, and model fitting}

This study aims to define the functional relationship among scan rate, temperature, number of cycles, $\mathrm{pH}$ sensitivity, and IrOx coating thickness by RSM with CCD. Design-Expert version 6.0.6 (StatEase, Inc., USA) was used to generate the regression model as well as perform statistical and data analyses. The variables in this study included three numerical factors: scan rate (X1), temperature (X2), and number of cycles (X3). The three independent variables and experimental design levels are shown in Table 1. Twenty experimental runs were determined from CCD, including eight factorial points, six axial points, and six centre points. The axial distance $(\alpha)$ from the centre point was set at 0.5 in coded units. The two-factor interaction model (2FI) to predict the optimal point can be written as follows:

$$
\mathrm{Y}=\mathrm{Xo}_{0}+\sum_{i-1}^{k} \mathrm{X}_{i} X_{i}+\sum_{i<j} \mathrm{X}_{i j} X_{i} X_{j}+\varepsilon_{\alpha}
$$

where $\mathrm{Y}$ represents the response ( $\mathrm{pH}$ sensitivity and $\mathrm{IrOx}$ coating thickness), and $\mathrm{y}_{0}, \mathrm{Y}_{\mathrm{i}}$, denote the constant coefficient and the first linear coefficient, respectively. yij is the linear model coefficient, $\mathrm{Xi}$ and $\mathrm{Xj}$ are the coded independent variables, and $\varepsilon$ is the standard error. Statistical "p" values less than 0.0500 were used to indicate the significant factors of the model. 


\section{Results and discussion}

\subsection{Fabrication of $\mathrm{pH}$ electrodes}

The FESEM images in Figure 1 reveal that the electrodeposited layer becomes more prone to cracking as the number of cycles increases, leading to enhanced $\mathrm{IrO}_{\mathrm{x}}$ thickness. All fabricated electrodes became crack-free after fabrication. Cracks result from layer dehydration in high-vacuum FESEM. This phenomenon clearly indicates that dehydration occurs more slowly in thicker layers than in thinner layers. Thus, more cracks are observed under fast-drying high-vacuum FESEM conditions.

\subsection{Response surface methodology model equations}

In this study, RSM was used to design an experiment and investigate the effect of independent variables such as scan rate, temperature, and cycles on three responses: $\mathrm{IrO}_{\mathrm{x}}$ coating thickness, $\mathrm{pH}$ electrode sensitivity, and Cathodic Storage Charge Capacity $\left(\mathrm{CSC}_{\mathrm{C}}\right)$. The $\mathrm{RSM}$ result showed no significant relationship between the independent variables and $\mathrm{pH}$ electrode sensitivity, which is consistent with previous findings. $\mathrm{CSC}_{\mathrm{C}}$ is directly related to $\mathrm{IrO}_{\mathrm{x}}$ thickness; thus, the DOE software was solely used to examine coded experimental models equations of $\mathrm{IrO}_{\mathrm{x}}$ thickness and the interaction of the significant terms in the proposed model.

\subsubsection{Coded experimental model equations for oxide thickness}

The DOE software suggested a 2FI model considering the adjusted R-squared and the predicted Rsquared rather than other models. The regression model for $\mathrm{IrO}_{\mathrm{x}}$ thickness, in coded factors, is shown by the following $2 F I$ model equation:

$$
H=931.20-(511.59 A)+(386.71 B)+(403.76 C)-(98.63 A B)-(433.63 A C)+(35.37 B C)
$$

where $\mathrm{H}$ is the $\mathrm{IrO}_{\mathrm{X}}$ thickness $(\mathrm{nm}) . \mathrm{A}, \mathrm{B}$, and $\mathrm{C}$ represent the scan rate, temperature, and number of cycles, respectively. According to equation 5, the predicted $\mathrm{R}^{2}$ of 0.2651 is not as close to the adjusted $\mathrm{R}^{2}$ of 0.8911 as normally expected. This discrepancy suggests a large block effect or a problem with this model.

To address this problem, insignificant terms (AB and $\mathrm{BC}$ ) should be removed from the proposed model to obtain an improved equation. The improved equation in terms of coded factors after removing the insignificant terms is shown as follows:

$$
H=+931.20-(511.59 A)+(386.71 B)+(403.76 C)-(433.62 A C)
$$

According to equation 3, the predicted $\mathrm{R}^{2}$ of 0.7444 is in agreement with the adjusted $\mathrm{R}^{2}$ of 0.8897 . Adequate precision measures the signal-to-noise ratio. A ratio greater than 4 is acceptable. The adequate precision ratio of 26.438 indicates an adequate signal. Therefore, this model can be used to navigate the design space. As indicated in Eq. (3), the main effects of temperature and number of cycles correlate positively with the $\mathrm{IrO}_{\mathrm{X}}$ thickness. The coefficients for scan rate (A) and number of cycles (C) are larger than the coefficients for temperature, indicating that parameters (A) and (B) have a more significant effect on the $\mathrm{IrO}_{\mathrm{X}}$ thickness than parameter $(\mathrm{B})$.

Figure 2 shows the predicted result obtained from Eq. (3), which is in agreement with the experimental data, thereby indicating the reliability of the improved model for predicting $\mathrm{IrO}_{\mathrm{X}}$ coating thicknesses under various electrodeposition conditions. An acceptable correlation-to- linear regression fit was also obtained, with an $\mathrm{R}^{2}$ of 0.9255 for $\mathrm{IrO}_{\mathrm{X}}$ thickness. 


\subsubsection{Interactions between independent variables}

Three-dimensional response surface plots and two-dimensional contour plots are presented in Figure 5 , which introduces the effects of number of cycles and scan rate at minimum and maximum temperatures on $\mathrm{IrO}_{\mathrm{X}}$ coating thickness $(\mathrm{nm})$. These types of plot depict the effect of the interaction of two factors on the response at a time, which can demonstrate sensitivities with variable changes.

In the present study, increasing the number of cycles at low scan rates leads to an increase in deposit thickness. This enhancement in $\mathrm{IrO}_{\mathrm{X}}$ thickness becomes more apparent at high temperatures, as indicated in Figures 5(a) and (b). Increase the number of cycles and decreasing the scan rate significantly affect the thickness of the electrodeposited layer.

\section{Conclusion}

This work demonstrates the efficiency of response surface methodology in modelling and investigating the effect of various factors on $\mathrm{IrO}_{\mathrm{X}}$ electrodeposition. Statistical results showed that the significant effect of scan rate on the $\mathrm{IrO}_{\mathrm{X}}$ layers is higher than those of temperature and number of cycles. The results of the electrochemical experiment indicated that all fabricated electrodes were conductive and reduced the impedance of stainless steel electrode. The $\mathrm{CV}$ result showed that $\mathrm{CSC}_{\mathrm{C}}$ increased with an increase in the electrodeposited layer. However, the FESEM images revealed that cracks in electrodeposited layer also increased with an increase in $\mathrm{IrO}_{\mathrm{X}}$ thickness.

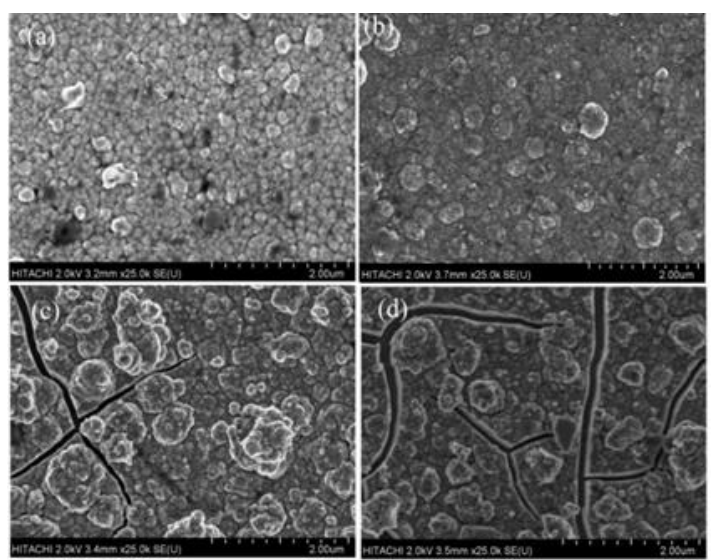

Figure 1. FESEM images of IrOx electrodeposited layer with an increasing number of cycles. (a) 100, (b) 200, (c) 400, and (d) 500

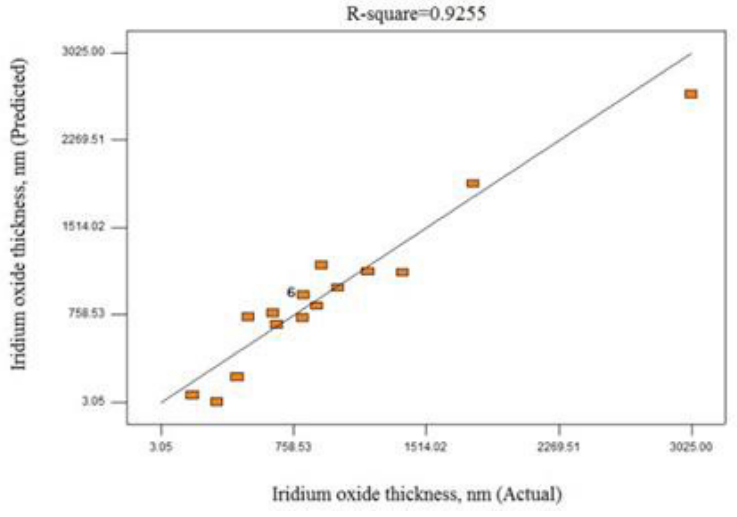

Figure 2. Actual versus predicted response plot of the IrOx coating thickness 

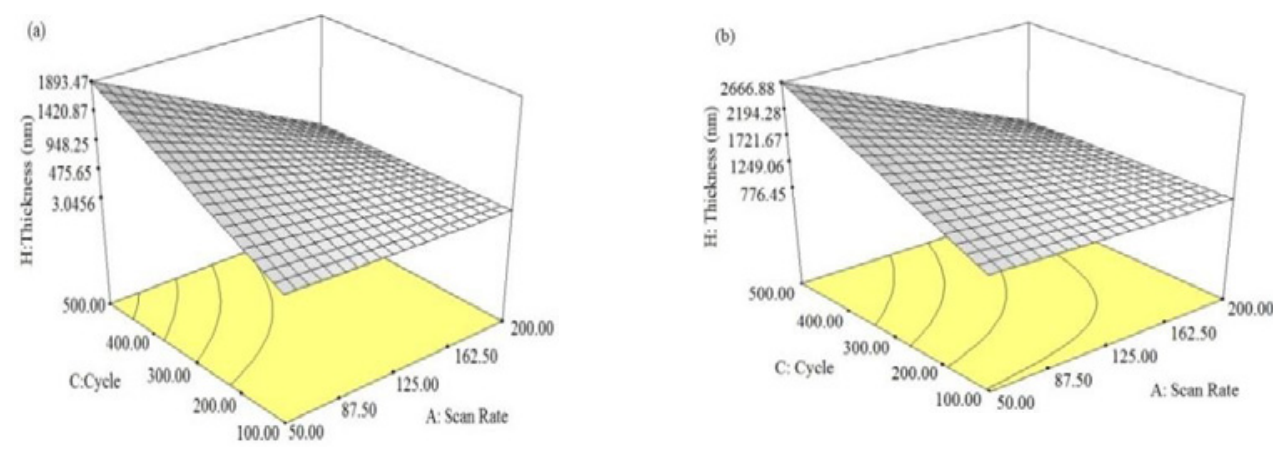

Figure 3. Response surface and contour plot of $\mathrm{IrO}_{\mathrm{x}}$ coating thickness $(\mathrm{nm})$ as a function of the number of cycles and scan rate at minimum and maximum temperatures: (a) $25^{\circ} \mathrm{C}$ (b) $65^{\circ} \mathrm{C}$.

\section{References}

1. Kurzweil, P., Sensors. 9, 4955-4985 (2009).

2. Ryynänen, T., Nurminen, K., Hämäläinen, J., Leskelä, M. \& Lekkala, J., Procedia Engineering. 5, 548-551 (2010).

3. Kakooei, S., Ismail, M.C. \& Ari-Wahjoedi, B., International Journal of Material Science Innovations. 1, 62-72 (2013).

4. Marzouk, S.A.M., Anal Chem. 75, 1258-1266 (2003).

5. Kakooei, S., Ismail, M.C. \& Wahjoedi, B.A., Int. J. Electrochem. Sci. 8, 3290-3301 (2013).

6. Yamanaka, K., Japanese journal of applied physics. 28, 632-637 (1989). 